\title{
Long-lived phonons
}

\author{
J. Qu and W. E. Bron \\ Department of Physics, University of California, Irvine, Irvine, California 92717 \\ (Received 23 February 1993; revised manuscript received 17 June 1993)
}

\begin{abstract}
We have demonstrated that long-lived transverse acoustic phonons exist, and that they can be observed as a product of three-particle anharmonic decay of polaritons in GaP. The long-lived phonons are found in a strongly dispersive region of the Brillouin zone and possess a frequency of $\sim 118 \mathrm{~cm}^{-1}$ and a lifetime of $66.5 \pm 5 \mathrm{~ns}$.
\end{abstract}

We have previously demonstrated that optical phonons and polaritons in simple compound semiconductors, e.g., $\mathrm{GaP}$, possess dephasing times in the picosecond and femtosecond time scales. ${ }^{1}$ In high-purity materials the source of the dephasing is dominated by three-particle anharmonic interactions. Others ${ }^{2}$ have demonstrated that longitudinal acoustic (LA) and transverse acoustic (TA) phonons decay with rates which vary with the inverse fifth power of the phonon frequency. The decay times of LA phonons can also be very short. ${ }^{3}$

In contrast to the short lifetimes of optical, LA, and some TA phonons, Orbach and Vredevoe $^{3}$ have noted that if the lowest (in energy) TA phonon branch is dispersive, then the TA phonons cannot readily decay by three-particle interactions and simultaneously obey energy and (crystal) momentum conservation. As a consequence, these phonons should possess anomalously long lifetimes.

At the time of Orbach and Vredevoe's paper, the existence of such long-lived phonons would have verified the simple, but critical, tenet that the phonon branches are in general dispersive and, in the absence of other scattering mechanisms, that the lowest-order phononphonon anharmonic interaction, plus energy and momentum conservation, govern phonon lifetimes. Although most of these tenets have since been verified by other means, none of these means have unambiguously measured the lifetime of long-lived phonons. Since long-lived phonons are a necessary ingredient in phonon bottlenecking ${ }^{4}$ and phonon breakdown, ${ }^{5}$ there has been much interest in attempts to demonstrate their existence.

We now demonstrate that long-lived TA phonons which have been generated near the $X$-point of the Brillouin zone of $\mathrm{GaP}$ possess a lifetime of $66.5 \pm 5 \mathrm{~ns}$. We start with our recent observation ${ }^{1}$ that in $\mathrm{GaP}$ a polariton $(\pi)$ of frequency $\omega_{\pi} \approx 341 \mathrm{~cm}^{-1}$ can decay into two LA phonons, each of $\omega_{\mathrm{LA}} \approx 172 \pm 26 \mathrm{~cm}^{-1}$ (i.e., $\omega_{\mathrm{LA}}=\omega_{\pi} / 2$ ), with equal, but oppositely signed, wave vectors. However, and more importantly here, we have also observed that a polariton with $\omega_{\pi} \approx 354 \mathrm{~cm}^{-1}$ can decay into one LA phonon with $\omega_{\mathrm{LA}} \approx 236 \pm 16 \mathrm{~cm}^{-1}$ and one TA phonon with $\omega_{\mathrm{TA}} \approx 118 \pm 16 \mathrm{~cm}^{-1}$. These latter results should be compared to the phonon frequencies observed at the $X$-point through neutron scattering; ${ }^{6}$ namely, $\omega_{\mathrm{LA}}(X)=249 \pm 4 \mathrm{~cm}^{-1}$ and $\omega_{\mathrm{TA}}(-X)=107 \pm 3 \mathrm{~cm}^{-1}$.
Thus symbolically the dominant decay process for $\omega_{\pi} \approx 354 \mathrm{~cm}^{-1}$ polaritons is

$$
\begin{aligned}
\pi\left(354 \mathrm{~cm}^{-1}, k \sim 2000 \mathrm{~cm}^{-1}\right) \\
\quad \Longrightarrow \operatorname{LA}\left(236 \mathrm{~cm}^{-1}, \sim \pm X\right)+\mathrm{TA}\left(118 \mathrm{~cm}^{-1}, \sim \mp X\right) .
\end{aligned}
$$

We will now demonstrate that these " $X$-point" TA phonons are the source of the long-lived acoustic phonons. The polaritons discussed above were generated through coherent Raman excitation ${ }^{7}$ (CRE) and their dephasing was measured through time resolved coherent anti-Stokes Raman scattering (TR-CARS). ${ }^{7}$ The variation of the polariton dephasing times with ambient temperatures serves to determine that the LA and TA phonons are generated near the $X$-point of the Brillouin zone due to the decay of the polaritons. ${ }^{1}$

Unfortunately, the acoustic phonons in GaP are not Raman active, at least in first order, and cannot be detected by TR-CARS. Thus we add to the TR-CARS technique ${ }^{1}$ vibronic sideband phonon spectroscopy (VSPS), ${ }^{8}$ which one of the authors developed some time ago for the detection of heat pulses. This technique is adapted to the present experiment in order to monitor the population of polaritons, and LA and TA acoustic phonons. In VSPS, electronic transitions (zero phonon line), localized at some optically active impurity, are modulated by phonons or polaritons which can couple to the transition. As a result Stokes and anti-Stokes sidebands arise in much the same manner as its radio frequency, FM, counterpart. In the presence of phonons, an anti-Stokes sideband appears whose amplitude is proportional to the phonon occupation number ${ }^{8}$ and whose spectral separation from the zero phonon line is a function of the phonon spectral distribution. In this way the concentration of polaritons and LA and TA phonons are monitored as a function of a delayable laser pulse which triggers the VSPS luminescence.

The laser system used in the experiment contains two pulsed nitrogen lasers, one of which, together with two Rhodamine $6 \mathrm{G}$ tunable dye lasers, is used to produce two "yellow" laser beams with a linewidth of $3 \mathrm{~cm}^{-1}$, pulse durations of $5 \mathrm{~ns}$, and $70 \mu \mathrm{J}$ energy per pulse. The two "yellow" output beams are tuned such that their frequen- 
cy difference equals $354 \mathrm{~cm}^{-1}$ or $341 \mathrm{~cm}^{-1}$. The beam directions are adjusted to conserve crystal momentum when a polariton is generated through CRE. A second pulsed nitrogen laser is used to pump a dye laser using coumarin 485, which in turn, is used to generate the "green" VSPS zero-phonon luminescence at $535 \mathrm{~nm}$. The source of the zero-phonon luminescence is known ${ }^{9}$ to result from an excitation of a bound exciton associated with nitrogen impurities in GaP. The luminescence can be delayed electronically over a range of $\sim 100 \mathrm{~ns}$, with a resolution of $\sim 5 \mathrm{~ns}$. The diameter of the focal spot of the various laser beams inside the sample is $100 \mu \mathrm{m}$. The output of the VSPS signal is collected and digitally stored in an optical multichannel analyzer (OMA). The delay between the "yellow" lasers and the "green" lasers is separately monitored through a digital oscilloscope. The detection range of the OMA is set to cover the frequency range from that of polaritons to that of the TA phonons and to block out the very strong, zero-phonon luminescence. The entire system is calibrated using standard reference lines. The sample is held at $4-5 \mathrm{~K}$ in order to obtain a sideband signal with narrow spectral width and with the best signal-to-noise ratio. The multiple beam overlap volume is located as near as possible to the entrance surface of the crystal to minimize the strong absorption at the "green" frequency. It should be noted that the laser system used in the present experiment differs from that used to determine the decay channels of coherent polaritons in GaP. ${ }^{1}$ Thus the results reported here are new independently obtained results.

The sample used in this experiment is the same highpurity sample used in Refs. 1 and 10, to measure, among other parameters, the third-order nonlinear electronic susceptibility, $\chi^{(3)}$. The imaginary component of $\chi^{(3)}$ reflects the amount of two-photon absorption. It was found to be very small under laser excitations similar to those used in the present experiment. See Ref. 10 and Table III therein. ${ }^{11}$

With $\omega_{\pi} \sim 341 \mathrm{~cm}^{-1}$, the only observed decay products should be two LA phonons with frequency $\omega_{\mathrm{LA}}=\omega_{\pi} / 2 .^{1}$ This result is indeed observed and is displayed in the top panel of Fig. 1. Here, we are more directly interested in $\omega_{\pi} \sim 354 \mathrm{~cm}^{-1}$, which leads to the " $X$-point" decay discussed earlier. The corresponding experimental results are indicated in Fig. 1, which is a display of the VSPS signal amplitude vs the phonon (and polariton) frequencies for various delays of the probe pulses. The highest panel of Fig. 1 corresponds to $\Delta t=0$ and indeed indicates the presence of $\omega_{\pi} \sim 354 \mathrm{~cm}^{-1}$, the $\mathrm{LA}(X)$ phonon at $\sim 236$ $\mathrm{cm}^{-1}$ and the TA $(X)$ phonon at $\sim 118 \mathrm{~cm}^{-1}$. Note that within a probe delay of $<10 \mathrm{~ns}$, both the polariton and the LA phonons have decayed. This result is consistent with their expected short lifetimes.

On the other hand, the VSPS signal associated with the TA $(X)$ phonon does indeed persist to longer delay times. Since this is, potentially, the long-lived phonon, we examine more carefully the meaning of the apparent slow decay rate as implied by the rest of Fig. 1. We note immediately that the signal at $118 \mathrm{~cm}^{-1}$ is not present for $\omega_{\pi} \sim 341 \mathrm{~cm}^{-1}$ nor is it for the case when $\omega_{\pi}$ is slightly detuned from $354 \mathrm{~cm}^{-1}$. These observations rule out the

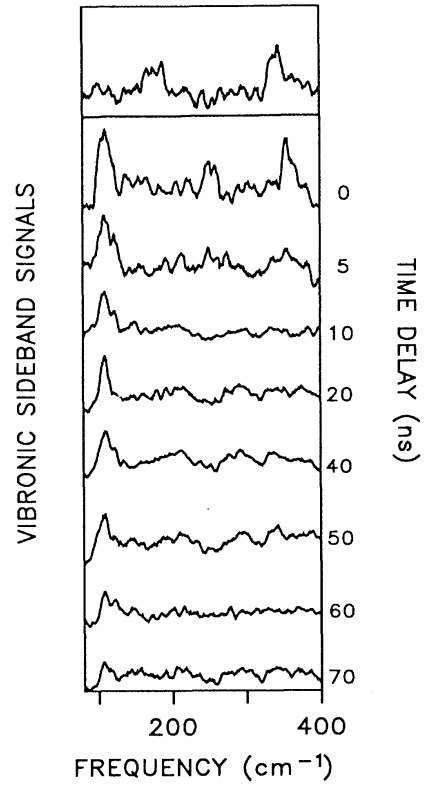

FIG. 1. Vibronic sideband amplitude, in arbitrary units as a function of phonon (and polariton) frequency for various delay times.

possibility that the signal at $\sim 118 \mathrm{~cm}^{-1}$ arises accidently from some stray luminescence. Furthermore, as a starting point of each series of observations, we carefully aligned all of the laser beams, as to their polarization and crystal orientation to satisfy the full CARS requirements. ${ }^{10}$ It would, accordingly, be strange indeed if the luminescence caused by any two-photon absorption of either of the pump beams just happened to correspond to the full CARS requirements, and cause a stray luminescence at $188 \mathrm{~cm}^{-1}$. We conclude, accordingly, that we observe the decay of TA phonons near the $X$-point of GaP.

The VSPS signal is a temporal (and spectral) convolution of the zero-phonon luminescence and the phonon sideband signal. The luminescent lifetime of the bound exciton is $38 \mathrm{~ns},{ }^{8}$ over which we integrate by setting the OMA gate to $150 \mathrm{~ns}$. We assume that the decay of the zero-phonon line and that of the TA sideband is exponential, such that

$$
f(t)-\left\{\begin{array}{l}
e^{-t / \tau_{1}} \\
0
\end{array}, g(t)=\left\{\begin{array}{l}
e^{-t / \tau_{2}}, \quad t \geq 0 \\
0, \quad t<0
\end{array}\right.\right.
$$

and

$$
f(t, \Delta t)=\left\{\begin{array}{l}
f(t) e^{-\Delta t / \tau_{1}}, \quad t \geq 0 \\
0, \quad t<0
\end{array}\right.
$$

in which $f(t)$ and $g(t)$ describe the decay of the TA phonons and the luminescent state, respectively; $f(t, \Delta t)$ describes the TA phonon decay after a delay of $\Delta t$, and $\tau_{1}$ and $\tau_{2}$ are the lifetimes of the TA phonon and the luminescent state, respectively. The evolution of the magnitude of the TA sideband is then of the form 


$$
\begin{aligned}
F(\Delta t) \int_{0}^{T} f(t, \Delta t) \otimes g(t) d t \\
=e^{-\Delta t / \tau_{1}} \int_{0}^{T} f(t) \otimes g(t) d t=C e^{-\Delta t / \tau_{1}}+B
\end{aligned}
$$

in which $\otimes$ refers to the convolution of $f(t)$ with $g(t), C$ is a contrast determined by the integration over the gate duration $T$ of the OMA, and $B$ is the magnitude of the signal background. We have performed fits to Eq. (2) for the three parameters $C, \tau_{1}$, and $B$. the resulting leastsquares fit (solid line) of the signal amplitude, as a function of the delay time, is compared to the data in Fig. 2. The error bars on the data points are the uncertainties in the background signals. The best fit of the lifetime of $\mathrm{TA}(X)$ phonons in $\mathrm{GaP}$ is $66.5 \pm 5 \mathrm{~ns}$.

Before proceeding, it is necessary to discuss the energy and wave vector of the long-lived phonon relative to that of the $X$-point. The published dispersion relations ${ }^{6}$ of $\mathrm{GaP}$ are too extensive to reproduce here. These relations, obtained theoretically and experimentally, are available only at room temperature. On the other hand VSPS almost always is conducted at liquid-helium temperatures to avoid thermal broadening of the zero phonon line and that of the vibronic sideband. A direct comparison is, therefore, not possible. If we take into account the change in frequency observed for the $354 \mathrm{~cm}^{-1}$ polariton with ambient temperature, and assume the phonon near the $X$-point to have a similar frequency shift, then the energy of the TA $(X)$ phonon $\sim 116 \pm 16 \mathrm{~cm}^{-1}$ (at RT). This range includes the TA phonon directly at the $X$-point $\left(107 \pm 3 \mathrm{~cm}^{-1}\right)$ but also includes a possible range of $132-100 \mathrm{~cm}^{-1}$. If the TA phonon exists directly at the $X$-point, then three-particle decay is kinematically possible along the $\Sigma$ direction. However, in order to satisfy energy and momentum conservation one of the decay products would need to have an energy higher than the TA $(X)$ phonon and one to be lower. Neither one of the $\Sigma$ phonons are observed in the vibronic spectra. We assume that the long-lived phonon is near, but not at the $X$-point.

It is well known ${ }^{13}$ that the ratio of amplitude of the anti-Stokes vibronic sideband associated with some vibrational state and its Stokes counterpart, yields the occupation probability of that state. We have measured the ratio for the TA $(X)$ phonon to be $5.5 \pm 1 \times 10^{-5}$. We have also calculated the occupation probability from the energy transferred from the two yellow lasers to the lattice. For this purpose, we have adapted our earlier analysis for the case of LA phonons in GaP. (See Sec. IV of Ref. 14.) We find values of $10^{-4}-10^{-3}$ depending on the parameters used to determine the overlap volume of the two "yellow" lasers. Both results suggest that under the present conditions, the $\mathrm{TA}(X)$ phonon state is too weakly occupied to play a role in phonon bottlenecking or pho-

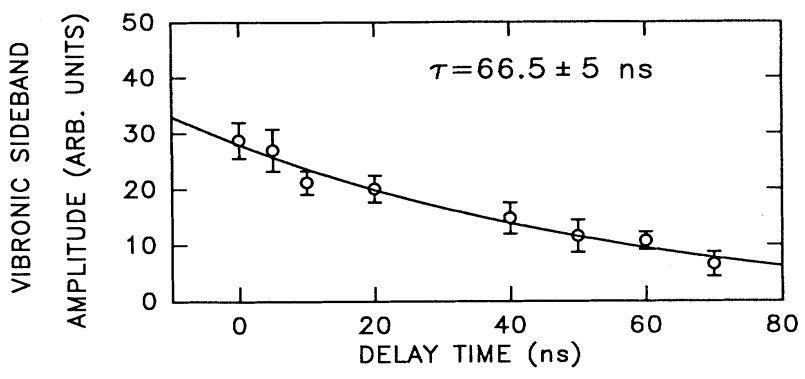

FIG. 2. Vibronic sideband amplitude as a function of delay time. The solid line is an analytic fit to the data (symbols).

non breakdown.

It would be interesting to compare the observed TA $(X)$ phonon lifetime to theoretically obtained values for $\tau_{1}$. It is clear that the direct decay of the $\mathrm{TA}(X)$ phonon into two TA phonons is not possible, due to the requirement of energy and momentum conservation. The decay of the $\mathrm{TA}(X)$ phonon is possible through third-order processes, by an absorption of a thermal TA phonon to create a LA phonon of higher frequency. The latter phonon can decay and conserve energy and momentum. Orbach and Vredevoe [see Eq. (3) of Ref. 3] have calculated the lifetime of a $30 \mathrm{~cm}^{-1}$ TA phonon in a generic solid at $T=2$ $\mathrm{K}$, and find a lifetime of $\sim 38 \times 10^{-6} \mathrm{~s}$ (and $10^{3} \mathrm{~s}$ for a fourth-order decay). We have scaled the phonon frequency (to $118 \mathrm{~cm}^{-1}$ ) and the ambient temperature to $4 \mathrm{~K}$, and find a lifetime of $\sim 40 \mu$ s for the third-order decay, and $0.03 \mathrm{~s}$ for the fourth-order process. In the calculation we have left out any variation of the group velocities. We also ignore (as do Orbach and Vredevoe) any anisotropy in the dispersion relations, or the presence of lattice imperfections which can cause elastic mode conversion from TA into LA phonons, which latter phonons can decay more rapidly than the $\mathrm{TA}(X)$ phonon.

We have demonstrated that a long-lived species of TA phonons exists with a wave vector near the $X$-point of GaP. The lifetime of these phonons is $66.5 \pm 5$ ns. This lifetime is much longer than that observed for LO $(\sim 26$ ps), polaritons ( $\sim 0.1 \mathrm{ps})$, and LA phonons. ${ }^{3,15}$ The relatively long life results from restrictions due to energy and momentum conservation. A theoretical comparison will have to await an analysis of phonon lifetimes in the strongly dispersive region of realistic anisotropic Brillouin zones, and in the presence of lattice imperfections.

The authors acknowledge support through NSF Grant No. DMR-89-13289.
${ }^{1}$ J. Kuhl and W. E. Bron, Solid State Commun. 49, 935 (1984); T. Juhasz and W. E. Bron, Phys. Rev. Lett. 63, 2385 (1989). Earlier work on polaritons appears in S. Ushioda, J. D. Mullen, and M. J. Delaney, Phys. Rev. B 8, 4634 (1989).

${ }^{2}$ K. F. Renk, in Nonequilibrium Phonons in Nonmetallic Crystals, edited by W. Eisenmenger and A. A. Kaplyanskii (Elsevier Science, Amsterdam, 1986), p. 277.
${ }^{3}$ R. Orbach and L. A. Vredevoe, Physics 1, 91 (1964).

${ }^{4}$ See, for example, R. Bray, K. T. Tsen, and K. Wan, in Phonon Scattering in Condensed Matter, edited by W. Eisenmenger, K. Lassmann, and S. Döttinger (Springer, Berlin, 1984), p. 121. See also R. G. Ulbrich, in Nonequilibrium Phonon Dynamics, edited by W. E. Bron (Plenum, New York, 1985), p. 101 , and references cited therein. 
${ }^{5}$ R. Orbach, IEEE Trans. Sonics Ultrason. SU-14, 140 (1967).

${ }^{6} \mathrm{H}$. Bilz and W. Kress, Phonon Dispersion Relations in Insulators (Springer, Berlin, 1979), p. 104.

${ }^{7}$ See, for example, W. E. Bron, in Dynamical Properties of Solids, edited by G. K. Horton and A. A. Maradudin (Elsevier Science, Amsterdam, 1990), p. 1.

${ }^{8}$ See, for example, W. E. Bron, Rep. Prog. Phys. 43, 301 (1980).

${ }^{9}$ D. G. Thomas, M. Gerhenson, and J. J. Hopfield, Phys. Rev. 131, 2347 (1963); D. G. Thomas and J. J. Hopfield, ibid. 150, 580 (1966).

${ }^{10}$ W. E. Bron, J. Kuhl, and B. K. Rhee, Phys. Rev. B 34, 6961 (1986).
${ }^{11}$ We do have a series of doped samples (Ref. 12), one of which was used at an early state of the present experiment. No VSPS signal was observed with this sample, which is, however, known to be dissipative.

${ }^{12}$ W. E. Bron, S. Mehta, J. Kuhl, and M. Klingenstein, Phys. Rev. B 39, 12642 (1989).

${ }^{13}$ See, for example, W. E. Bron and W. Grill, Phys. Rev. B 16, 5303 (1977).

${ }^{14}$ S. Mehta, T. Juhasz, and W. E. Bron, Phys. Rev. B 45, 209 (1992).

${ }^{15}$ S. Tamura, Phys. Rev. B 31, 2574 (1985). 